\title{
Many-body interactions and Rashba splitting of the surface state on $\mathrm{Cu}(110)$
}

\author{
J. Jiang, ${ }^{1}$ S. S. Tsirkin, $, 2,3,{ }^{*}$ K. Shimada,,${ }^{1} \dagger$ H. Iwasawa, ${ }^{1}$ M. Arita, ${ }^{1}$ H. Anzai, ${ }^{1}$ H. Namatame, ${ }^{1}$ M. Taniguchi, ${ }^{1,4}$ \\ I. Yu. Sklyadneva, ${ }^{2,3,5,6}$ R. Heid, ${ }^{5}$ K.-P. Bohnen, ${ }^{5}$ P. M. Echenique, ${ }^{2,7}$ and E. V. Chulkov ${ }^{2,3,7}$ \\ ${ }^{1}$ Hiroshima Synchrotron Radiation Center, Hiroshima University, Kagamiyama 2-313, Higashi-Hiroshima 739-0046, Japan \\ ${ }^{2}$ Donostia International Physics Center (DIPC), 20018 San Sebastián/Donostia, Basque Country, Spain \\ ${ }^{3}$ Tomsk State University, 634050, Tomsk, Russian Federation \\ ${ }^{4}$ Graduate School of Science, Hiroshima University, Kagamiyama 1-3-1, Higashi-Hiroshima 739-8526, Japan \\ ${ }^{5}$ Karlsruher Institut für Technologie, Institut für Festkörperphysik, D-76021 Karlsruhe, Germany \\ ${ }^{6}$ Institute of Strength Physics and Materials Science, pr. Academicheskii 2/1, 634021, Tomsk, Russian Federation \\ ${ }^{7}$ Departamento de Física de Materiales UPVIEHU, Centro de Física de Materiales CFM-MPC and Centro Mixto CSIC-UPV/EHU, 20080
}

San Sebastián/Donostia, Basque Country, Spain

(Received 20 June 2013; published 5 February 2014)

\begin{abstract}
Using high-resolution angle-resolved photoemission spectroscopy, we elucidate the Rashba splitting of $\Delta k_{F}=$ $0.003 \AA^{-1}$ near the Fermi level $\left(E_{F}\right)$ in the Shockley surface state of $\mathrm{Cu}(110)$ at the $\overline{\mathrm{Y}}$ point of the surface Brillouin zone. The observed energy-band dispersion exhibits a kink structure at $\sim-20 \mathrm{meV}$, which is a clear indication of band renormalization caused by an electron-phonon interaction. The electron-phonon coupling parameter is found to be $\lambda_{e p}=0.17 \pm 0.02$ based on the experimentally obtained real part of the self-energy. First-principle calculations yield $\lambda_{e p}=0.160$ and $\Delta k_{F}=0.004 \AA^{-1}$ at $E_{F}$, which are fully consistent with the experimental results. In addition, the contributions of the electron-electron and electron-phonon interactions to the linewidth of the surface state at the $\overline{\mathrm{Y}}$ point are experimentally determined to be $\Gamma_{e e} \sim 9 \mathrm{meV}$ and $\Gamma_{e p} \sim 7 \mathrm{meV}$, respectively. We demonstrate that the Rashba splitting must be resolved by photoemission line-shape analysis for an accurate determination of the electron self-energy and coupling parameters.
\end{abstract}

DOI: 10.1103/PhysRevB.89.085404

PACS number(s): 73.20.At, 73.22.Lp, 79.60.-i

\section{INTRODUCTION}

Shockley-type surface states on metal surfaces have been widely used as a testing system to study various interactions [1-3], such as the electron-electron interaction (EEI) [4], the electron-phonon interaction (EPI) [5], and the electron-defect interaction (EDI) [6]. Many-body interactions in solids influence a variety of physical properties, ranging from electrical transport to superconductivity [7]. In particular, these interactions cause the renormalization of excited quasiparticles, which manifests itself via phenomena such as a reduced lifetime [3,4] and kinks in quasiparticle dispersion [5,8], and play an important role in catalysis and photochemistry $[9,10]$.

High-resolution angle-resolved photoemission spectroscopy (ARPES) is a powerful tool for studying many-body interactions on solid surfaces $[5,8,11]$. From the quantitative line-shape analysis of ARPES spectra, one can experimentally evaluate both the imaginary part of the self-energy $(\operatorname{Im} \Sigma)$, which is related to the lifetime broadening, and the real part of the self-energy $(\operatorname{Re} \Sigma)$, which represents an energy shift from the noninteracting band [5,8,11]. However, it is still challenging to experimentally determine the self-energy because an accurate measurement requires high energy and momentum resolutions.

The total linewidth $\Gamma_{\text {tot }}$ of an electronic state contains contributions from the EEI $\left(\Gamma_{e e}\right)$, EPI $\left(\Gamma_{e p}\right)$, and EDI $\left(\Gamma_{e d}\right)$. However, because of the broken translational symmetry at the surface, spin-orbit coupling (SOC) induces spin splitting of the surface states (the Rashba effect [Ref. [12]]). When the spin

\footnotetext{
*tsirkinss@gmail.com

†kshimada@hiroshima-u.ac.jp
}

splitting of an electronic state caused by the Rashba effect (Rashba splitting) is comparable to the linewidth broadening due to the EDI, it is not possible to evaluate contributions from the EEI and EPI except for the high-symmetry points where no Rashba splitting exists. Therefore, it is crucial to determine the magnitude of the Rashba splitting for spectroscopic studies of many-body interactions.

The Rashba effect has been extensively studied in surface states that are mostly centered at the $\bar{\Gamma}$ point of the surface Brillouin zone (SBZ) in many heavy elements, such as $\mathrm{Au}(111)$ [2,13,14], $\mathrm{Ag} / \mathrm{Au}(111)$ [15], and low-index surfaces of $\mathrm{Bi}[16,17]$, as well as in the surface alloys $\mathrm{Sb} / \mathrm{Ag}(111)$ [18], Bi/Ag(111) [19,20], and Bi/Cu(111) [21]. Because SOC is weaker in elements with a smaller nuclear charge, it has been difficult to detect the Rashba splitting in light elements. Just recently the splitting has been reported for the $\mathrm{Cu}(111)$ surface [22].

On the (110) surfaces of $\mathrm{Cu}, \mathrm{Ag}$, and $\mathrm{Au}$, there exist Shockley-type surface states at the $\overline{\mathrm{Y}}$ point of the SBZ [see Figs. 1(a) and 1(b)]. However, the band renormalization in the surface states at the $\bar{Y}$ point remains unexplored. While first-principle electronic-structure calculations predict the existence of Rashba-type spin splitting in the surface state of $\mathrm{Au}(110)[24,25]$, the splitting on the (110) surfaces of noble metals has not yet been observed experimentally even for heavy metals such as $\mathrm{Au}(110)$ [25].

In this study, we perform a comprehensive experimental and theoretical investigation of the many-body interactions and Rashba splitting of an occupied surface state of $\mathrm{Cu}(110)$. The electron self-energy derived from the EPI and the contributions of the EPI, EEI, and EDI to the linewidth are experimentally determined via a line-shape analysis of ARPES data at low temperature. We calculated the magnitudes of the EPI 
(a)

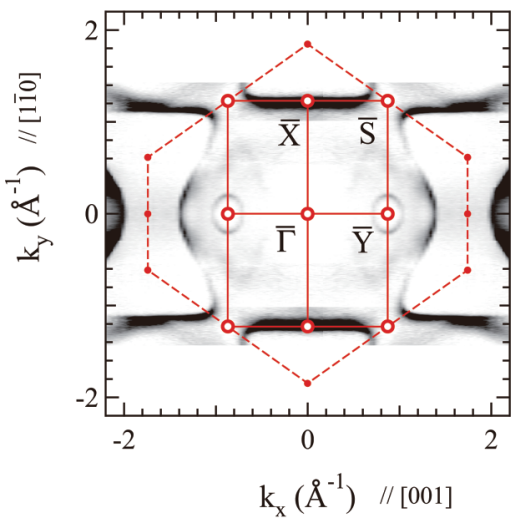

(b)

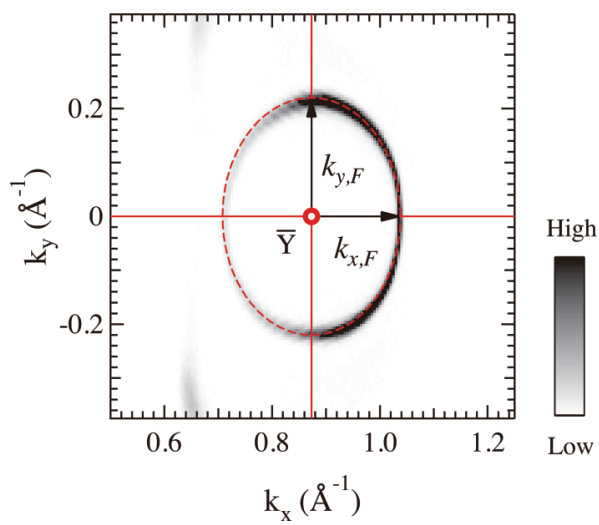

FIG. 1. (Color online) (a) A Fermi surface mapping of the $\mathrm{Cu}(110)$ surface at $h v=91$ $\mathrm{eV}$ and $10 \mathrm{~K}$ (Ref. [23]). Whereas the solid lines indicate the surface Brillouin zone with high-symmetry points, the dashed lines show the bulk Brillouin zone on the $\Gamma X U L K$ plane. (b) Fermi surface mapping of the $\mathrm{Cu}(110)$ surface state near the $\bar{\Gamma}$ point at $h v=9 \mathrm{eV}$ and $10 \mathrm{~K}$. $k_{x, F}=0.18 \AA^{-1}$ and $k_{y, F}=0.22 \AA^{-1}$ indicate the measured Fermi wave vectors in the $\overline{\Gamma Y}$ and $\overline{\Gamma X}$ directions, respectively. and Rashba splitting from first principles, and found that the theoretical results exhibit excellent agreement with the experimental ones. We demonstrate that despite the weakness of the SOC in $\mathrm{Cu}$, the finite Rashba splitting must be considered to precisely evaluate the strength of many-body interactions; therefore, high energy and momentum resolution is especially crucial for the ARPES study of many-body interactions.

\section{EXPERIMENTAL}

High-resolution ARPES experiments were performed on the BL-9A beamline of a compact electron-storage ring (HiSOR) at Hiroshima University [26]. The data were obtained using the angular mode of a hemispherical electron-energy analyzer (R4000, VG-Scienta, Sweden). The total energy resolution was set to $\Delta E=5 \mathrm{meV}$ for a photon energy of $h v=9 \mathrm{eV}$. Tunable synchrotron radiation allowed us to optimize the incident photon energy. The angular resolution was $\Delta \theta=0.1^{\circ}$, which provides a momentum resolution of $\Delta k_{\|}=0.002 \AA^{-1}$. The sample temperature was set to $10 \mathrm{~K}$ during the ARPES measurements.

Single-crystalline $\mathrm{Cu}(110)(99.999 \%)$ was cleaned by repeated cycles of $\mathrm{Ar}^{+}$sputtering $(1.5 \mathrm{kV})$ and annealing $\left(420^{\circ} \mathrm{C}\right)$ in an ultrahigh-vacuum chamber. The levels of impurities such as $\mathrm{C}, \mathrm{O}$, and $\mathrm{S}$ on the surface were below the detection limit of Auger electron spectroscopy. Sharp $1 \times 1$ lowenergy electron-diffraction spots demonstrated long-range order and an atomically clean sample surface. The quality of the surface was confirmed by the linewidth of the energy distribution curve (EDC) at the $\bar{Y}$ point, which is sensitive to the contribution from the EDI.

\section{DENSITY-FUNCTIONAL THEORY CALCULATION}

Density-functional theory (DFT) calculations of the Rashba splitting for the occupied surface state were performed using the VASP code [27,28], which employs the projectoraugmented wave (PAW) method [29,30] and the generalized gradient approximation (GGA). The surface was represented by 41-layer slabs, which was sufficient to avoid any artificial splitting of the surface state due to interactions between opposite surfaces of the slab.

EPI calculations were performed using density-functional perturbation theory [31] with a mixed-basis pseudopotential approach [32] and a norm-conserving pseudopotential [33] with nonlinear core corrections. The exchange-correlation energy functional was evaluated within the GGA. In this case, the surface was represented by periodically repeating 19-layer $\mathrm{Cu}(110)$ slabs separated by six atomic layers of vacuum.

\section{RESULTS AND DISCUSSION}

Figure 2(a) shows the ARPES intensity plot of the $\mathrm{Cu}(110)$ surface state along the $\bar{\Gamma} \mathrm{Y}$ direction, which exhibits parabolic dispersion. By fitting the momentum distribution curves (MDCs) and EDCs to a Lorentzian superimposed on a linear background, we determined the peak positions, which are plotted as open circles in Fig. 2(c). At the $\bar{Y}$ point $\left(k_{\bar{Y}}=0.873 \AA^{-1}\right)$, the energy of the surface state is $\omega_{0}=-466 \mathrm{meV}$ at $10 \mathrm{~K}$, which agrees with previous studies giving $\omega_{0}=-470 \mathrm{meV}$ at $140 \mathrm{~K}$ (Ref. [1]), and $\omega_{0}=-450 \mathrm{meV}$ at room temperature (Ref. [34]). However, previous study estimated $\omega_{0}$ at $0 \mathrm{~K}$ as $\omega_{0}=-510 \mathrm{meV}$ based on the linear fit to the temperature dependence of $\omega_{0}$ from $630 \mathrm{~K}$ down to $140 \mathrm{~K}$ [1]. Present study indicates that the temperature dependence is much smaller below $140 \mathrm{~K}$ because $\omega_{0}$ at $10 \mathrm{~K}$ and at $140 \mathrm{~K}$ is almost identical.

By fitting the observed EDC at the $\bar{Y}$ point to a Lorentzian [Fig. 2(b)], the total width was determined to be $\Gamma_{\text {tot }}=26 \mathrm{meV}$, which includes three independent contributions: $\Gamma_{\text {tot }}=\Gamma_{e p}+$ $\Gamma_{e e}+\Gamma_{e d}$. The total lifetime broadening is $\sim 50 \%$ of the previously reported value $\left[\Gamma_{\text {tot }}=48 \pm 6 \mathrm{meV}\right.$ at $140 \mathrm{~K}$ (Ref. [1])]; this difference is primarily attributable to the reduced thermal broadening and the smaller contribution from $\Gamma_{e d}$ in this study.

The calculations performed using the VASP code yielded a surface band energy of $-540 \mathrm{meV}$ at the $\overline{\mathrm{Y}}$ point, which is slightly lower than the experimental result. For a convenient comparison of the band dispersions, we shifted the theoretical dispersions in Fig. 2(c) upward to coincide with the experimental ones at the $\bar{Y}$ point. Far from the $\bar{Y}$ point, the surface-state dispersion splits into two branches: an inner branch $\omega_{\text {in }}(k)$ and an outer branch $\omega_{\text {out }}(k)$. Near the $\bar{Y}$ point $\left(\left|k-k_{\overline{\mathrm{Y}}}\right|<0.06 \AA^{-1}\right)$, this dispersion can be fitted using the Rashba model:

$$
\omega_{\text {in }(\text { out })}(k)=\omega_{0}+\frac{\hbar^{2}\left(k-k_{\overline{\mathrm{Y}}}\right)^{2}}{2 m^{*}} \pm \alpha_{R}\left(k-k_{\overline{\mathrm{Y}}}\right),
$$




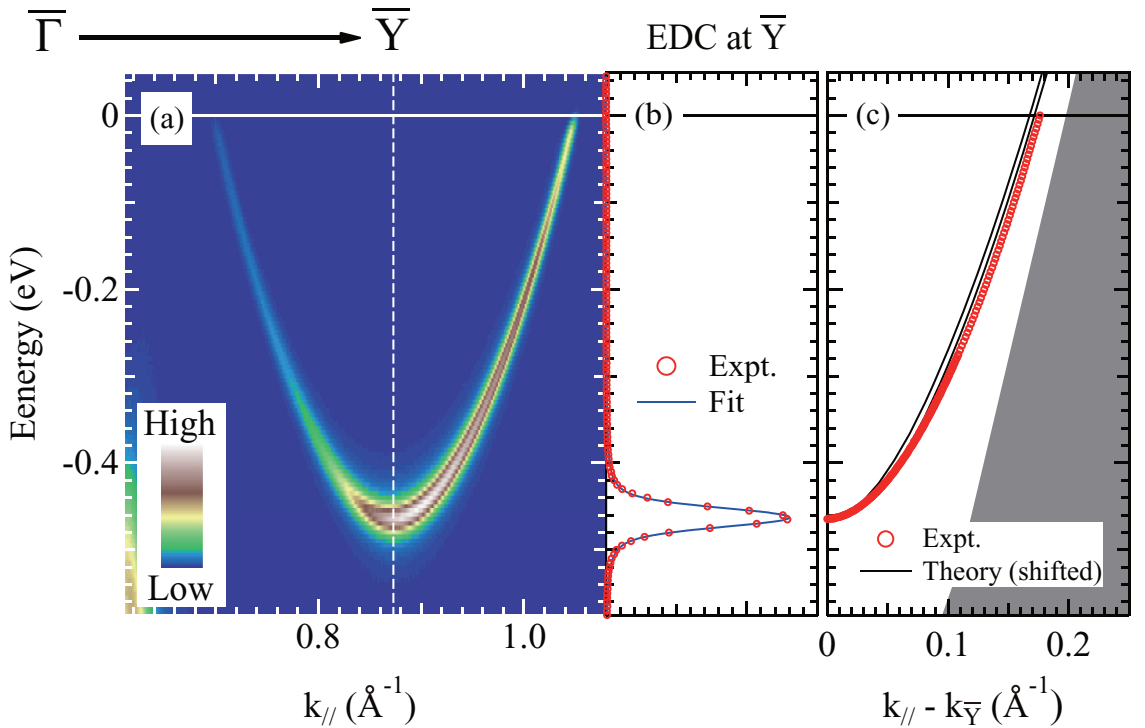

FIG. 2. (Color online) (a) An ARPES intensity plot of the $\mathrm{Cu}(110)$ surface state over a wide energy range for $h v=9 \mathrm{eV}$ and $10 \mathrm{~K}$. (b) The EDC at the $\overline{\mathrm{Y}}$ point and its fit using a Lorentzian. (c) Band points obtained via MDC and EDC analyses (the open circles). The solid lines indicate the calculated dispersion of the Rashbasplit surface state along the $\overline{\Gamma Y}$ direction, which has been shifted upward to coincide with the experimental results at the $\overline{\mathrm{Y}}$ point. The gray area indicates the calculated projection of the bulk states onto the SBZ. where $\alpha_{R}=\frac{\hbar^{2} k_{R}}{m^{*}}=0.12 \mathrm{eV} \AA$ is the Rashba parameter, $k_{R}=0.003 \AA^{-1}, m^{*}=0.19 m_{e}$ ( $m_{e}$ : electron mass), and $k_{\overline{\mathrm{Y}}}$ represents the wave number for the $\bar{Y}$ point. Near the Fermi level $\left(E_{F}\right)$, Eq. (1) does not give a good approximation of the dispersion because of its nonparabolic character. While the wave-number splitting of the surface state at the $\bar{Y}$ point is $\Delta k_{\overline{\mathrm{Y}}}=2 k_{R}=0.006 \AA^{-1}$, this value decreases as $\left|k-k_{\overline{\mathrm{Y}}}\right|$ increases and is only $\Delta k_{F}=0.004 \AA^{-1}$ at the Fermi wave number $k_{F}=\left|k-k_{\bar{Y}}\right| \approx 0.17 \AA^{-1}$. Note that the measured Fermi wave number along the $\overline{\Gamma \bar{Y}}$ direction was evaluated to be $k_{x, F}=\left|k-k_{\bar{Y}}\right| \approx 0.18 \AA^{-1}$ [Fig. 1(b)] consistent with our calculation. Based on the measured Fermi surface, the carrier density of the surface state for each spin direction is calculated to be $n=k_{x, F} k_{y, F} / 4 \pi=3.2 \times 10^{13}$ electrons $/ \mathrm{cm}^{2}$, which is $\sim 86 \%$ of the corresponding carrier density for $\mathrm{Cu}(111)$ (Ref. [2]). The difference appears to be reasonable because the $L$ gap of the bulk Fermi surface projected on the (110) SBZ is compressed by $\sim 82 \%$ along the $\overline{\Gamma Y}$ direction compared with that projected on the (111) SBZ.

In the mixed-basis pseudopotential calculation, we obtained a surface-state energy of $-465 \mathrm{meV}$ at the $\bar{Y}$ point in perfect agreement with the ARPES data $(-466 \mathrm{meV})$. The calculated electron-phonon coupling parameter $\lambda_{e p}=0.155$ at the $\bar{Y}$ point is smaller than both the previously reported experimental value of $\lambda=0.23 \pm 0.02$ (Ref. [1]) and the value found in previous model calculations, namely, $\lambda=0.24$ (Refs. [35,36]). Furthermore, the calculated electron-phonon contribution to the linewidth $\Gamma_{e p}=7.7 \mathrm{meV}$ at $T=0 \mathrm{~K}$ is slightly smaller than the contribution predicted by the model calculations, $\Gamma_{e p}=9.6 \mathrm{meV}$ (Ref. [36]). At $E_{F}$, the present calculation gives the electron-phonon coupling parameter of $\lambda_{e p}=0.160$, which is close to the value at the $\overline{\mathrm{Y}}$ point $\left(\lambda_{e p}=0.155\right)$.

We should note that SOC is not included in the EPI calculation. Our calculations indicate that over $90 \%$ of $\lambda_{e p}$ is determined by the transitions of holes from the surface state to bulk states. Because the splitting is rather small for the surface state under consideration, the rates of phononmediated scattering from the surface state to bulk states are nearly equal for the inner and outer branches of the surface band. Thus, the coupling parameters should be practically identical for the two split branches. The inclusion of SOC in the calculation of the electron-phonon coupling would require extremely time-consuming computations and would not allow us to use slabs of sufficient thickness.

Figure 3(b) shows a high-resolution ARPES intensity plot of the $\mathrm{Cu}(110)$ surface state near $E_{F}$. The peak splitting is clearly discernible in the MDC [Fig. 3(a)]. We used two Voigt functions to fit the MDCs to quantitatively analyze the spectral shape. We fixed the Gaussian width, which represents the momentum resolution of $\Delta k_{\|}=0.002 \AA^{-1}$, and we optimized Lorentzian width for these two peaks. The splitting at $E_{F}$ was estimated to be $\Delta k_{F}=0.003 \AA^{-1}$, which is close to our calculated value of $\Delta k_{F}=0.004 \AA^{-1}$. Note that the Rashba splitting in $\mathrm{Cu}$ is one order of magnitude smaller than the corresponding splitting in the $\mathrm{Au}(111)$ Shockley state, $\Delta k_{F}=0.023-0.026 \AA^{-1}$ (Refs. [13,14]). The splitting is even smaller than the Rashba splitting in $\mathrm{Cu}(111), \Delta k_{F} \sim$ $0.006 \AA^{-1}$ (Ref. [22]).

In Fig. 3(a), the Lorentzian width (the full width at half maximum) of the inner (outer) -branch peak was determined to be $\delta k=0.0023(0.0024) \AA^{-1}$. The mean-free path of the surface state may be evaluated by $\bar{l}=1 / \delta k$ (Ref. [37]) $\sim 430 \AA$, that is, $\sim 120 a$, where $a=3.61 \AA$ is the lattice parameter of $\mathrm{Cu}$ (Ref. [38]).

For the surface states, the linewidth due to the final-state broadening is negligible [39], and the lifetime broadening derived from the EPI and EEI is $\sim 0$ at $E_{F}(\omega=0)$ at low temperature $[7,8,40]$. Therefore, the lifetime broadening at $E_{F}$ at $10 \mathrm{~K}$ should originate mainly from the EDI, where $\Gamma_{e d}=2|\operatorname{Im} \Sigma(0)|=\left.\frac{\partial \omega}{\partial k}\right|_{\omega=0} \delta k=10 \mathrm{meV}$, which is assumed to be independent of $\omega$ and $k$. Based on the ARPES results, the gradient of the band dispersion was evaluated as $\left.\frac{\partial \omega}{\partial k}\right|_{\omega=0}=$ $\hbar v_{F}=4.2 \times 10^{3} \mathrm{meV} \AA$, where $v_{F}$ is the Fermi velocity. The lifetime of the surface state at $E_{F}$ is, therefore, evaluated to be $\tau=\hbar / \Gamma_{e d} \sim 6.5 \times 10^{-14} \mathrm{~s}$ at $10 \mathrm{~K}$. The mobility of the electrons in the surface state may be evaluated as $\mu=e \tau / m^{*} \sim$ $600 \mathrm{~cm}^{2} /(\mathrm{V} \mathrm{s})$ at $10 \mathrm{~K}$. While the mobility is larger than that for the surface state on $\mathrm{Al}(100)\left[\mu=4.4 \mathrm{~cm}^{2} /(\mathrm{V} \mathrm{s})\right.$ at $10 \mathrm{~K}$ 

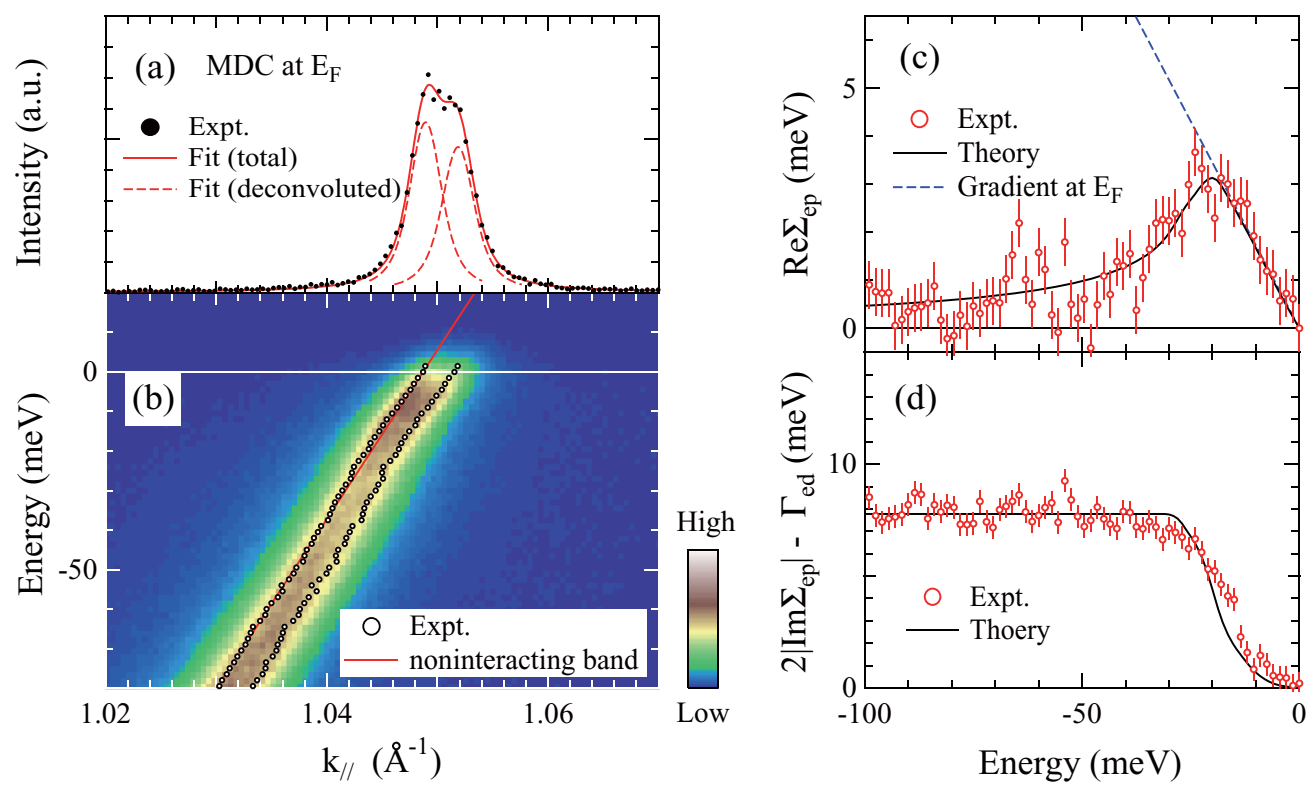

FIG. 3. (Color online) (a) The MDC at $E_{F}$ (the dots) fitted with two Voigt functions. The solid and dashed lines indicate the total and deconvoluted fitting curves, respectively. (b) The ARPES intensity plot of the $\mathrm{Cu}(110)$ surface state with the Rashba splitting for $h v=9$ eV and $10 \mathrm{~K}$. The open circles indicate the band points determined from the MDC peak positions, and the solid red line depicts the assumed noninteracting band for the evaluation of $\operatorname{Re} \Sigma_{e p}$. (c) The experimentally obtained Re $\Sigma_{e p}$ (the open circles) and the theoretical Re $\Sigma_{e p}$ (the solid line). The dashed line indicates the gradient of the experimental Re $\Sigma_{e p}$ at $E_{F}$, which was used to evaluate $\lambda_{e p}$. (d) The experimentally obtained $2|\operatorname{Im} \Sigma|-\Gamma_{e d}$ (the open circles) and the theoretical $2\left|\operatorname{Im} \Sigma_{e p}\right|$ (the solid line). The energy dependence of the lifetime broadening near $E_{F}$ is mainly described by $2\left|\operatorname{Im} \Sigma_{e p}\right|$.

(Ref. [8])], it is much smaller than that of high mobility twodimensional electron gas existing in, e.g., $\mathrm{GaAs}_{\mathrm{Al}} \mathrm{Al}_{x} \mathrm{Ga}_{1-x} \mathrm{As}$ interface with $\mu=14 \times 10^{6} \mathrm{~cm}^{2} /(\mathrm{V} \mathrm{s})$ at $20 \mathrm{mK}$ [41].

The experimental band dispersion of the surface state was determined from the MDC peak positions [the open circles in Fig. 3(b)]. A kink in the energy-band dispersion exists at $\sim-20 \mathrm{meV}$, which is consistent with the energy scale of the Debye energy for $\mathrm{Cu}, k_{B} \Theta_{D}=27 \mathrm{meV}\left(\Theta_{D}=315 \mathrm{~K}\right)$ [7].

To quantitatively characterize the EPI, we performed self-energy analyses for the inner branch of the Rashbasplit bands with a stronger intensity assuming that the many-body interactions in the Rashba-split bands are practically identical [42].

Figure 3(c) shows the experimental and theoretical real part of the self-energy $\operatorname{Re} \Sigma_{e p}$ due to the EPI. The experimental $\operatorname{Re} \Sigma_{e p}$ was evaluated from $\operatorname{Re} \Sigma_{e p}(\omega)=\omega-\omega_{k}^{0}$, where $\omega_{k}^{0}$ is the noninteracting band given by a parabolic fit to the experimental band dispersion over a wide energy range. One can confirm that the theoretical $\operatorname{Re} \Sigma_{e p}$ [the solid line in Fig. 3(c)] quantitatively reproduces the experimental peak position at $\sim-20 \mathrm{meV}$ and the maximum energy shift of $\sim 3 \mathrm{meV}$.

The electron-phonon coupling parameter can be evaluated from the gradient of the experimental $\operatorname{Re} \Sigma_{e p}$ at $E_{F}$. The dashed line in Fig. 3(c) shows the gradient of the experimental $\operatorname{Re} \Sigma_{e p}$ obtained from a least-squares fit in the energy range of $-20 \mathrm{meV}<\omega<0 \mathrm{meV}$. The coupling parameter was found to be $\lambda_{e p}=-\left.\frac{\partial \operatorname{Re} \Sigma_{e p}}{\partial \omega}\right|_{\omega=0}=0.17 \pm 0.02$, which is in agreement with the result of our DFT calculation, namely, $\lambda_{e p}=0.160$. Note that the previous model calculation gave $\lambda_{e p}=0.24$ for the $\mathrm{Cu}(110)$ surface [36], which is slightly larger than the value given by the DFT calculation. We also found that the electron-phonon coupling parameter for the (110) surface is close to the value obtained for the (111) surface $\lambda_{e p}=0.16$ (Ref. [22]). In the case of the $\mathrm{Cu}(111)$ the model calculation gave a coupling parameter of $\lambda_{e p}=0.16$ (Ref. [40]) in agreement with the experiment.

Figure 3(d) shows the experimentally determined energydependent part of the linewidth from the formula $2|\operatorname{Im} \Sigma(\omega)|-$ $\Gamma_{e d}=\frac{\partial \omega}{\partial k} \delta k-\Gamma_{e d}$. Excellent agreement is observed again between the theoretical $2\left|\operatorname{Im} \Sigma_{e p}(\omega)\right|$ and the experimental $2|\operatorname{Im} \Sigma(\omega)|-\Gamma_{e d}$ values, which is a clear indication of the negligible contribution from the EEI in this energy range. The measured broadening due to the EPI at $10 \mathrm{~K}$ is $\Gamma_{e p} \sim 7 \mathrm{meV}$ for an energy range of $k_{B} \Theta_{D}(\sim 27 \mathrm{meV})$ $<|\omega|<100 \mathrm{meV}$, consistent with the value obtained from our calculation: $\Gamma_{e p}=7.7 \mathrm{meV}$. Thus, the lifetime broadening due to the EEI should be $\Gamma_{e e}=\Gamma_{\text {tot }}-\Gamma_{e d}-\Gamma_{e p} \sim 9 \mathrm{meV}$ at the $\bar{Y}$ point, which is significantly smaller than the broadening predicted by model calculations, $\Gamma_{e e}=21 \mathrm{meV}$ (Ref. [43]). We note that an accurate experimental evaluation of the lifetime broadening contributes to the critical assessment and fundamental development of many-body theory.

Finally, we emphasize the importance of including Rashba splitting in line-shape analysis to quantitatively evaluate the electron-phonon coupling parameter and electron self-energy. To demonstrate this point, we present ARPES results from a $\mathrm{Cu}(110)$ surface with a higher defect concentration in Figs. 4(a)-4(d). The Rashba splitting cannot be resolved because of the large contribution from $\Gamma_{e d}$ [Figs. 4(a) and 4(b)]. The EDC width at the $\overline{\mathrm{Y}}$ point is as large as $\Gamma_{\text {tot }}=34 \mathrm{meV}$. In Fig. 4(a), the MDC width at $E_{F}$ represented by the Lorentzian width is $\delta k=0.022 \AA^{-1}$, which is almost one order 

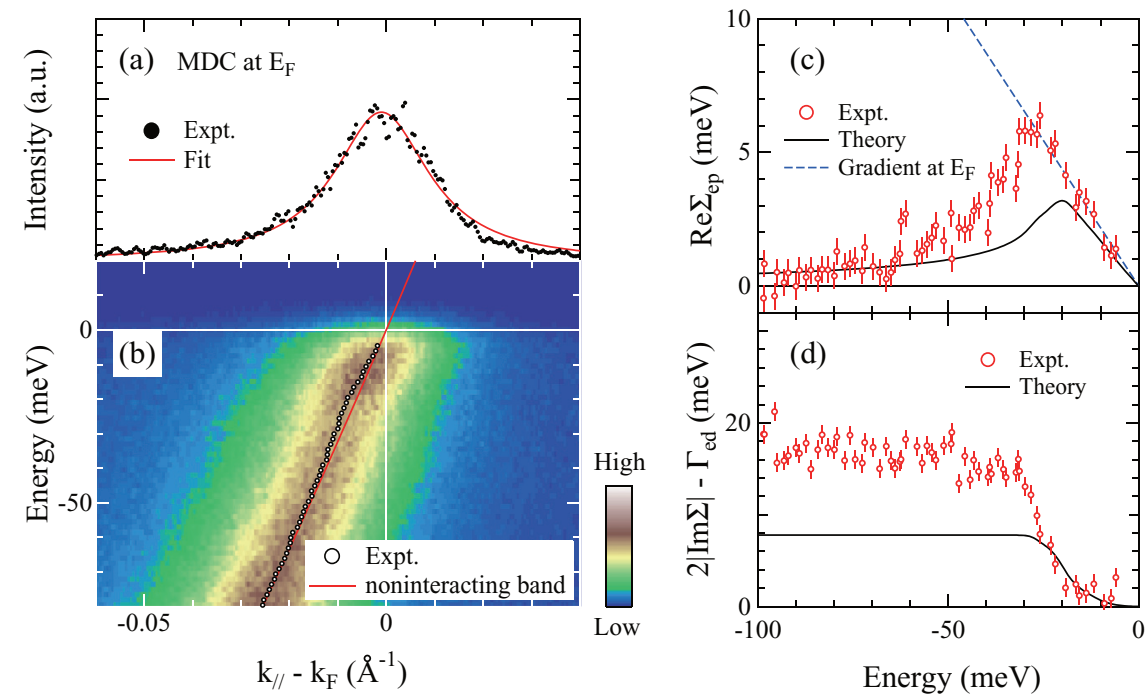

of magnitude larger than the results presented in Fig. 3(a). The experimentally determined $\operatorname{Re} \Sigma_{e p}(\omega)$ peak is located at $\sim-30 \mathrm{meV}$ [Fig. 4(c)], whereas the theoretical one is located at $\sim-20 \mathrm{meV}$. The electron-phonon coupling parameter was determined to be $\lambda=0.22$ using the gradient of the experimental $\operatorname{Re} \Sigma_{e p}(\omega)$ in Fig. 4(c), which is sufficiently larger than the theoretical value $\lambda=0.160$ and the experimental result $\lambda=0.17$ with the Rashba splitting taken correctly into account. We consider that these deviations in the real part of the self-energy most likely arise from the energy dependence of the relative intensities and/or linewidths of the Rashba-split bands [42]. The experimental $2|\operatorname{Im} \Sigma(\omega)|-\Gamma_{e d}$ neglecting the Rashba splitting is about two times larger than the theoretical one in Fig. 4(d), yielding inaccurate information on the lifetime broadening of quasiparticles.

\section{CONCLUSION}

We have performed a comprehensive experimental and theoretical study on the electron self-energy due to the EPI and Rashba splitting in the Shockley surface state of $\mathrm{Cu}(110)$. The observed Rashba-type spin splitting near $E_{F}$ is $\Delta k_{F}=$ $0.003 \AA^{-1}$ in agreement with the theoretical calculation of $\Delta k_{F}=0.004 \AA^{-1}$. The magnitude of this splitting is one-half of the value $\Delta k_{F} \sim 0.006 \AA^{-1}$ obtained for the $\mathrm{Cu}(111)$ surface. A kink structure associated with the EPI exists in the energy-band dispersion at $\sim-20 \mathrm{meV}$. The values of the electron-phonon coupling parameter derived from ARPES $\left(\lambda_{e p}=0.17 \pm 0.02\right)$ and DFT calculations $\left(\lambda_{e p}=\right.$ $0.160)$ coincide well. The contributions of the EEI and EPI
FIG. 4. (Color online) Results obtained with a higher defect concentration at the surface. (a) The MDC at $E_{F}$ (the dots) fitted with a single Voigt function (the solid line). Note that the wave-number range is twice the range in Fig. 3. (b) An ARPES intensity plot of the $\mathrm{Cu}(110)$ surface state for $h v=9 \mathrm{eV}$ and $10 \mathrm{~K}$. The open circles indicate the band points determined from the MDC peak positions, and the solid red line indicates the assumed noninteracting band for the evaluation of $\operatorname{Re} \Sigma_{e p}$. (c) The experimentally obtained $\operatorname{Re} \Sigma_{e p}$ (the open circles) and the theoretical Re $\Sigma_{e p}$ (the solid line). The dashed line indicates the gradient of the experimental $\operatorname{Re} \Sigma_{e p}$ at $E_{F}$, which was used to evaluate $\lambda_{e p}$. (d) The experimentally obtained $2|\operatorname{Im} \Sigma|-\Gamma_{e d}$ (the open circles) and the theoretical $2\left|\operatorname{Im} \Sigma_{e p}\right|$ (the solid line).

to the linewidth of the surface state at the $\overline{\mathrm{Y}}$ point were experimentally determined to be $\Gamma_{e e} \sim 9 \mathrm{meV}$ and $\Gamma_{e p} \sim 7$ $\mathrm{meV}$, respectively. We have demonstrated the importance of resolving the Rashba splitting of surface states in ARPES line-shape analysis, even for light elements with weak SOC. These results improve our quantitative understanding of the magnitudes of many-body interactions and Rashba splitting at a surface, which provides a firm basis for advanced surface science. At present, it is difficult to directly resolve the spin direction of the surface state on $\mathrm{Cu}(110)$. However, we believe it would be possible in the future based on the rapid progress of the spin-resolved ARPES technique [44].

\section{ACKNOWLEDGMENTS}

This work was partially supported by a Grant-in-Aid for Scientific Research (Grants No. 22340103 and No. 25400376) from MEXT of Japan and by the Ministry of Education and Science of the Russian Federation (state task No. 2.8575.2013). We thank N-BARD, Hiroshima University for supplying liquid helium. The synchrotron radiation experiments were performed with the approval of HSRC (Proposal No. 12-A-30). We thank H. Hayashi, T. Horike, and Y. Nagata for their support during the experiments. In addition, we acknowledge the financial support of the University of Basque Country UPV/EHU (Grant No. GIC07-IT-366-07), the Departamento de Educación del Gobierno Vasco and the Spanish Ministerio de Ciencia e Innovación (Grant No. FIS2010-19609-C02-01). The SOC calculations were performed on the SKIF-Cyberia computer at Tomsk State University.

[1] P. Straube, F. Pforte, T. Michalke, K. Berge, A. Gerlach, and A. Goldmann, Phys. Rev. B 61, 14072 (2000), and references therein.

[2] F. Reinert, G. Nicolay, S. Schmidt, D. Ehm, and S. Hüfner, Phys. Rev. B 63, 115415 (2001), and references therein.

[3] P. M. Echenique, R. Berndt, E. V. Chulkov, Th. Fauster, A. Goldmann, and U. Höfer, Surf. Sci. Rep. 52, 219 (2004).
[4] E. V. Chulkov, V. M. Silkin, and M. Machado, Surf. Sci 482-485, 693 (2001).

[5] Ph. Hofmann, I. Yu. Sklyadneva, E. D. L. Rienks, and E. V. Chulkov, New J. Phys. 11, 125005 (2009); E. V. Chulkov, J. Kliewer, R. Berndt, V. M. Silkin, B. Hellsing, S. Crampin, and P. M. Echenique, Phys. Rev. B 68, 195422 (2003). 
[6] M. Fuglsang Jensen, T. K. Kim, S. Bengió, I. Yu. Sklyadneva, A. Leonardo, S. V. Eremeev, E. V. Chulkov, and Ph. Hofmann, Phys. Rev. B 75, 153404 (2007).

[7] G. Grimvall, The Electron-Phonon Interaction in Metals (NorthHolland, Amsterdam, 1981).

[8] J. Jiang, K. Shimada, H. Hayashi, H. Iwasawa, Y. Aiura, H. Namatame, and M. Taniguchi, Phys. Rev. B 84, 155124 (2011), and references therein.

[9] R. Haight, Surf. Sci. Rep. 21, 275 (1995).

[10] P. Saalfrank, Chem. Rev. 106, 4116 (2006).

[11] T. Valla, A. V. Fedorov, P. D. Johnson, and S. L. Hulbert, Phys. Rev. Lett. 83, 2085 (1999).

[12] Yu. A. Bychkov and E. I. Rashba, JETP Lett. 39, 78 (1984).

[13] S. LaShell, B. A. McDougall, and E. Jensen, Phys. Rev. Lett. 77, 3419 (1996).

[14] M. Hoesch, M. Muntwiler, V. N. Petrov, M. Hengsberger, L. Patthey, M. Shi, M. Falub, T. Greber, and J. Osterwalder, Phys. Rev. B 69, 241401(R) (2004).

[15] A. Nuber, J. Braun, F. Forster, J. Minár, F. Reinert, and H. Ebert, Phys. Rev. B 83, 165401 (2011)

[16] T. Hirahara, K. Miyamoto, I. Matsuda, T. Kadono, A. Kimura, T. Nagao, G. Bihlmayer, E. V. Chulkov, S. Qiao, K. Shimada, H. Namatame, M. Taniguchi, and S. Hasegawa, Phys. Rev. B 76, 153305 (2007).

[17] Yu. M. Koroteev, G. Bihlmayer, J. E. Gayone, E. V. Chulkov, S. Blügel, P. M. Echenique, and Ph. Hofmann, Phys. Rev. Lett. 93, 046403 (2004).

[18] L. Moreschini, A. Bendounan, I. Gierz, C. R. Ast, H. Mirhosseini, H. Höchst, K. Kern, J. Henk, A. Ernst, S. Ostanin, F. Reinert, and M. Grioni, Phys. Rev. B 79, 075424 (2009).

[19] G. Bihlmayer, S. Blügel, and E. V. Chulkov, Phys. Rev. B 75, 195414 (2007).

[20] C. R. Ast, J. Henk, A. Ernst, L. Moreschini, M. C. Falub, D. Pacilé, P. Bruno, K. Kern, and M. Grioni, Phys. Rev. Lett. 98, 186807 (2007).

[21] L. Moreschini, A. Bendounan, H. Bentmann, M. Assig, K. Kern, F. Reinert, J. Henk, C. R. Ast, and M. Grioni, Phys. Rev. B 80, 035438 (2009).

[22] A. Tamai, W. Meevasana, P. D. C. King, C. W. Nicholson, A. de la Torre, E. Rozbicki, and F. Baumberger, Phys. Rev. B 87, 075113 (2013).

[23] Y. Miura, Master's thesis, Hiroshima University, 2008. All the experiments were performed on the HiSOR BL-1. The energy and angular resolutions were set at $50 \mathrm{meV}$ and $0.3^{\circ}$, respectively.
[24] M. Nagano, A. Kodama, T. Shishidou, and T. Oguchi, J. Phys.: Condens. Matter 21, 064239 (2009).

[25] A. Nuber, M. Higashiguchi, F. Forster, P. Blaha, K. Shimada, and F. Reinert, Phys. Rev. B 78, 195412 (2008).

[26] M. Arita, K. Shimada, H. Namatame, and M. Taniguchi, Surf. Rev. Lett. 9, 535 (2002).

[27] G. Kresse and J. Hafner, Phys. Rev. B 48, 13115 (1993).

[28] G. Kresse and J. Furthmüller, Comput. Mater. Sci. 6, 15 (1996).

[29] P. E. Blöchl, Phys. Rev. B 50, 17953 (1994).

[30] G. Kresse and D. Joubert, Phys. Rev. B 59, 1758 (1999).

[31] S. Baroni, S. de Gironcoli, A. Dal Corso, and P. Giannozzi, Rev. Mod. Phys. 73, 515 (2001).

[32] R. Heid and K.-P. Bohnen, Phys. Rev. B 60, R3709 (1999).

[33] D. Vanderbilt, Phys. Rev. B 32, 8412 (1985).

[34] O. Zeybek, A. M. Davarpanah, and S. D. Barrett, Surf. Sci. 600, 5176 (2006).

[35] S. S. Tsirkin, S. V. Eremeev, and E. V. Chulkov, Surf. Sci. 604, 804 (2010).

[36] S. V. Eremeev, S. S. Tsirkin, and E. V. Chulkov, Phys. Rev. B 82, 035424 (2010).

[37] T. Valla, A. V. Fedorov, P. D. Johnson, B. O. Wells, S. L. Hulbert, Q. Li, G. D. Gu, and N. Koshizuka, Science 285, 2110 (1999).

[38] C. Kittel, Introduction to Solid State Physics, 6th ed. (Wiley, New York, 1986).

[39] N. V. Smith, P. Thiry, and Y. Petroff, Phys. Rev. B 47, 15476 (1993).

[40] A. Eiguren, B. Hellsing, F. Reinert, G. Nicolay, E. V. Chulkov, V. M. Silkin, S. Hüfner, and P. M. Echenique, Phys. Rev. Lett. 88, 066805 (2002); A. Eiguren, B. Hellsing, E. V. Chulkov, and P. M. Echenique, Phys. Rev. B 67, 235423 (2003).

[41] W. Pan, N. Masuhara, N. S. Sullivan, K. W. Baldwin, K. W. West, L. N. Pfeiffer, and D. C. Tsui, Phys. Rev. Lett. 106, 206806 (2011).

[42] I. A. Nechaev, M. F. Jensen, E. D. L. Rienks, V. M. Silkin, P. M. Echenique, E. V. Chulkov, and Ph. Hofmann, Phys. Rev. B 80, 113402 (2009).

[43] S. S. Tsirkin, S. V. Eremeev, and E. V. Chulkov, Phys. Rev. B 84, 115451 (2011).

[44] T. Okuda, K. Miyamaoto, H. Miyahara, K. Kuroda, A. Kimura, H. Namatame, and M. Taniguchi, Rev. Sci. Instrum. 82, 103302 (2011). 